\title{
BOUNDARY PROBLEMS LEADING TO ORTHOGONAL POLYNOMIALS IN SEVERAL VARIABLES
}

\author{
BY F. V. ATKINSON
}

Communicated by Peter D. Lax, December 7, 1962

1. Introduction. For polynomials in one variable it is well known that an equivalence holds between orthogonality on the real axis and recurrence relations of a certain form. If namely $\psi(x)$ is nondecreasing for real $x$, has an infinity of points of increase, and has finite power-moments of all orders, and polynomials $p_{n}(x)$, of degree $n=0,1,2, \cdots$ are formed so that

$$
\int_{-\infty}^{\infty} p_{r}(x) p_{s}(x) d \psi(x)=0(r \neq s), \neq 0(r=s)
$$

then there holds [1] a three-term recurrence formula

$$
p_{n+1}(x)+\left(a_{n} x+b_{n}\right) p_{n}(x)+p_{n-1}(x)=0, \quad a_{n}>0,
$$

where we take formally $p_{-1}(x)=0, p_{0}(x)=1$, and have incorporated suitable constant factors in the $p_{n}(x)$. Conversely [2] we may pass from (1.2) to the existence of at least one spectral function $\psi(x)$ with respect to which (1.1) holds. The position is much less clear in respect to orthogonal polynomials in several variables, for which work has been devoted mainly to the approach which starts with the orthogonality and to analogues of the classical polynomials. This field is surveyed in [3], where it is noted that there is a deficiency of results leading from recurrence relations to orthogonality.

The purpose of this note is to indicate a genuine extension of (1.2) in the direction of several variables, preserving the usual oscillatory and orthogonal properties. We conclude by giving the relation between the polynomials to be defined and certain determinants of ordinary orthogonal polynomials, considered in a recent series of papers by Karlin and McGregor [4]; in addition, we indicate the relation between this topic and the more general subject of simultaneous eigenvalue problems involving several parameters.

2. The recurrence relations. For a fixed integer $k \geqq 1$ we shall define $k$ sets of polynomials $p_{n r}(\lambda), r=1, \cdots, k$, of degree $n$ in the $k$ variables $\lambda_{1}, \cdots, \lambda_{k}$; here we shall write $\lambda$ for the column-matrix formed by $\lambda_{1}, \cdots, \lambda_{k}$. Denoting by $a_{n r}$ certain row-matrices of real coefficients $a_{n r 1}, \cdots, a_{n r k}$ and by $b_{n r}$ real scalars we define the $p_{n r}(\lambda)$ recursively by $p_{-1, r}(\lambda)=0, p_{0 r}(\lambda)=1$, and 


$$
p_{n+1, r}(\lambda)+\left(a_{n r} \lambda+b_{n r}\right) p_{n r}(\lambda)+p_{n-1, r}(\lambda)=0 .
$$

We write also boldface $n$ for a $k$-tuple of integers $\left(n_{1}, \cdots, n_{k}\right)$, and write $|\boldsymbol{n}|=\max \left(n_{1}, \cdots, n_{k}\right)$; in addition we use the notations

$$
p_{n}(\lambda)=p_{n_{1} 1}(\lambda) p_{n_{2} 2}(\lambda) \cdots p_{n_{k} k}(\lambda)
$$

and

$$
a_{n}=\operatorname{det}\left|a_{n_{r}, r}\right|_{r=1}^{k}=\operatorname{det}\left|a_{n_{r} r s}\right|_{r, s=1}^{k},
$$

the middle form consisting of row-matrices.

We start by propounding the finite-dimensional boundary problem in which, for some positive integral $m$, we have

$$
p_{m r}(\lambda)=0, \quad r=1, \cdots, k .
$$

The solutions of these $k$ simultaneous polynomial equations in $k$ variables will be the eigenvalues, an eigenvalue being a $k$-tuple of scalars. By a simple extension of the usual arguments we have then

THEOREM 1. Let, for all $\boldsymbol{n}$ with $|\boldsymbol{n}|<m$,

$$
a_{n}>0 \text {. }
$$

Then the boundary problem (2.1), (2.4) has only real eigenvalues; if $\lambda, \mu$ are distinct eigenvalues, then

$$
\sum_{|n|<m} a_{n} p_{n}(\lambda) p_{n}(\mu)=0 .
$$

For the proof we have the $k$ Christoffel-Darboux formulae

$$
p_{m r}(\lambda) p_{m-1, r}(\mu)-p_{m r}(\mu) p_{m-1, r}(\lambda)=\sum_{j=0}^{m-1} a_{j r}(\mu-\lambda) p_{j r}(\lambda) p_{j r}(\mu),
$$

for $r=1, \cdots, k$, for any column-matrices $\lambda, \mu$ this being proved as in $[1, \S 3.2]$. If now $\lambda, \mu$ are distinct eigenvalues, the left vanishes by (2.4), and eliminating $(\mu-\lambda)$ we have

$$
\operatorname{det}\left|\sum_{j=0}^{m-1} a_{j r} p_{j r}(\lambda) p_{j r}(\mu)\right|_{r=1}^{k}=0,
$$

where we recall that the $a_{j r}$ are row-matrices. Each row in this determinant is formed of the sum of $m$ row-matrices, and the determinant may thus be expanded as the sum of $m^{k}$ determinants, and this is none other than (2.6).

If now $\lambda$ were a complex eigenvalue, and $\mu$ were its complex con- 
jugate, (2.6) would yield a contradiction with (2.5), since at least one of the $p_{n}(\lambda)$ is not zero, namely that given by $n=(0, \cdots, 0)$.

3. The oscillation theorem. The orthogonality (2.6) is one of vectors of dimension $m^{k}$, from which it follows that there are at most $m^{k}$ such vectors, and so at most $m^{k}$ eigenvalues. To show that there are exactly $m^{k}$ such eigenvalues is the role of certain Sturmian considerations, which will be presented in detail elsewhere [5]. The function $p_{n r}(\lambda)$ defined by $(2.1)$ is to be completed to a piecewise linear function $p_{x r}(\lambda)$, coinciding with $p_{n r}(\lambda)$ when $x=n$, so that we may assess the number of zeros of $p_{x r}(\lambda)$ as $x$ increases from -1 to $m$. Denoting by $\nu$ a $k$-tuple of non-negative integers $\left(\nu_{1}, \cdots, \nu_{k}\right)$ we have

THEOREM 2. For each $\nu,|\nu|<m$, there is an eigenvalue $\lambda^{(\nu)}$ such that $p_{x r}\left(\lambda^{(v)}\right)$ exhibits just $\nu_{r}$ changes of sign as $x$ increases in $-1<x<m$.

The proof to be given in [5] concerns Jacobian arguments applied to certain phase variables, and is distinct from the argument given in [6] for the analogous situation for differential equations.

4. Orthogonality. We may now write (2.6) as a complete set of orthogonal relations

$$
\sum_{|n|<m} a_{n} p_{n}\left(\lambda^{\left(\nu^{\prime}\right)}\right) p_{n}\left(\lambda^{(\nu)}\right)=\rho^{(\nu)} \delta_{\nu \nu^{\prime}}
$$

where $\nu, \nu^{\prime}$ run through all $k$-tuples of non-negative integers less than $m, \delta_{\nu \nu^{\prime}}$ is the Kronecker symbol and the $\rho^{(\nu)}$ are positive. Passing to the dual orthogonality relations we have the orthogonality of the polynomials, or

THEOREM 3. The polynomials $p_{n}(\lambda),|n|<m$ are orthogonal according to

$$
\sum_{|\nu|<m} p_{n}\left(\lambda^{(\nu)}\right) p_{n^{\prime}}\left(\lambda^{(\nu)}\right) / \rho^{(\nu)}=\delta_{n n^{\prime}} / a_{n}
$$

This is an orthogonality for only a finite number of the polynomials. For this we rewrite (4.2) as a Stieltjes integral in $k$ dimensions, with respect to the $k$-dimensional spectral function

$$
\psi_{m}(\lambda)=\sum_{\lambda^{(v)} \leq \lambda} 1 / \rho^{(\nu)},
$$

the inequality $\lambda^{(v)} \leqq \lambda$ being interpreted componentwise. We may then rewrite $(4.2)$ as

$$
\int_{-\infty}^{\infty} p_{n}(\lambda) p_{n^{\prime}}(\lambda) d \psi_{m}(\lambda)=\delta_{n n^{\prime}} / a_{n}
$$

the measure $d \psi_{m}(\lambda)$ being understood as in [7, Chapter II]. 
Making now $m \rightarrow \infty$, we may conclude that there exists at least one limiting ( $k$-dimensional) spectral function $\psi(\lambda)$, bounded and ( $k$-dimensionally) nondecreasing, and such that

$$
\int_{-\infty}^{\infty} p_{n}(\lambda) p_{n^{\prime}}(\lambda) d \psi(\lambda)=\delta_{n n^{\prime}} / a_{n}
$$

for all $n$. For this purpose we need the Helly-Bray theorems, modified to deal with the case of polynomial integrands in $k$ dimensions. The uniqueness of the spectral function may be related, to some extent as in the usual case, to limit-point and limit-circle eventualities.

5. Symmetries. For any integral $q \geqq 0$ we now introduce the notation $\left(a_{q}\right)$ for the square matrix formed by the row matrices $a_{q_{1}}, \cdots, a_{q_{k}}$; this is to be distinguished from the determinant, with boldface suffix, defined in (2.3).

In considering possible symmetry properties of the $\left(a_{q}\right)$, let us first note the case in which the $\left(a_{q}\right)$ are all multiples of $E_{k}$, the $k$ th order unit matrix, and the $b_{q r}$ are independent of $r$. We have in this case a "separation of variables," the $p_{n r}(\lambda)$ are polynomials in the $\lambda_{r}$ separately, drawn from one and the same set of ordinary orthogonal polynomials. The $p_{n}(\lambda)$ given by (2.2) will be products of such polynomials, and the determinants of Karlin and McGregor will be linear combinations of such products. In what follows we describe a class of situations which include this.

We denote by $\Gamma$ the group of $k$-by- $k$ permutation matrices $S$ which commute with all the $\left(a_{q}\right), q=0,1, \ldots$; for simplicity we suppose also that the $b_{q r}$ are independent of $r$. In general, of course, $\Gamma$ will consist only of $E_{k}$, while in the case just described it will be the full symmetric group. The following is relevant to the case that $\Gamma$ is the symmetric group or a nontrivial subgroup.

We note the transformation formula

$$
p_{n}(S \lambda)=p_{S^{*} n}(\lambda)
$$

where the $k$-tuple of integers $n$ is treated as a column matrix, and $S^{*}$ is the Hermitean conjugate of $S$, which is the same as its inverse and its transpose, for a permutation matrix. For on replacing $\lambda$ by $S \lambda$ in (2.1) we have, with $b_{n}$ for $b_{n r}$,

$$
p_{n+1, r}(S \lambda)+\left(a_{n r} S \lambda+b_{n}\right) p_{n r}(S \lambda)+p_{n-1, r}(S \lambda)=0 .
$$

Let $S$ be put explicitly in the form $\left(\delta_{s, \sigma(r)}\right)_{r, s=1}^{k}$, this being the entry in the $r$ th row and $s$ th column, and $\sigma(1), \cdots, \sigma(k)$ being a permutation of $1, \cdots, k$. Since $\left(a_{n}\right) S=S\left(a_{n}\right), a_{n r} S$ is the $r$ th row in $S\left(a_{n}\right)$, 
which is found to be $a_{n \sigma(r)}$. Hence (5.2) is the same as the recurrence relation for $p_{n \sigma(r)}(\lambda)$, so that

$$
p_{n r}(S \lambda)=p_{n \sigma(r)}(\lambda) .
$$

We may then verify (5.1) by means of (2.2).

Let now $\chi(S)$ be a unitary representation of $\Gamma$ by matrices. In extension of the above-mentioned determinants of orthogonal polynomials we define the polynomials

$$
P_{n}(\lambda)=\sum_{S \in \Gamma} p_{n}(S \lambda) \chi(S)=\sum_{S \in \Gamma} p_{S^{*}{ }_{n}}(\lambda) \chi(S) .
$$

For any $T \in \Gamma$ we have then

$$
P_{n}(T \lambda)=\sum_{S \in \Gamma} p_{n}(S T \lambda) \chi\left(S T T^{-1}\right)=P_{n}(\lambda) \chi\left(T^{-1}\right) .
$$

We now set up orthogonality relations for the $P_{n}(\lambda)$. We recall that orthogonalities exist both of the type (4.1), with summation over $\boldsymbol{n}$, and of the type (4.2), with summation over $\lambda$, and again that they may be finite-dimensional as in (4.1)-(4.2) or infinite as in (4.4). Here we consider only the latter type. Assuming (4.4) we have

$$
\begin{aligned}
\int_{-\infty}^{\infty} P_{n}(\lambda) P_{n^{\prime}}^{*}(\lambda) d \psi(\lambda) & =\sum_{S} \sum_{S^{\prime}} \chi(S) \chi^{*}\left(S^{\prime}\right) \int_{-\infty}^{\infty} p_{S^{*} n}(\lambda) p_{S^{\prime *} n^{\prime}}(\lambda) d \psi(\lambda) \\
& =\sum_{\substack{S \\
\left(S^{*} n=S^{\prime \prime} n^{\prime}\right)}} \sum_{\substack{S^{\prime}\\
}\left(S S^{\prime-1}\right) / a_{S^{*} n} .}
\end{aligned}
$$

Suppose now that the equation $S^{*} n=S^{*} n^{\prime}$ has no solutions, that is to say, with $U=S^{*-1} S^{*}=S S^{-1}, n=U n^{\prime}, U \in \Gamma$ is insoluble. We may achieve this by confining $\boldsymbol{n}, \boldsymbol{n}^{\prime}$ to a fundamental region of the set of $\{\boldsymbol{n}\}$ with respect to $\Gamma$, and have then

$$
\int_{-\infty}^{\infty} P_{n}(\lambda) P_{n^{\prime}}(\lambda) d \psi(\lambda)=0
$$

excluding from consideration $\boldsymbol{n}$ which are invariant under some $U$, other than the identity. In the case when $\Gamma$ is the full symmetric group, a suitable region is given by $n_{1}<n_{2}<\cdots<n_{k}$.

Exploiting now the property, a consequence of (5.4),

$$
P_{n}(T \lambda) P_{n^{\prime}}^{*}(T \lambda)=P_{n}(\lambda) P_{n^{\prime}}^{*}(\lambda), \quad T \in \Gamma,
$$

we see that under certain conditions (5.5) may be replaced by an integral over a fundamental region of euclidean $k$-space with respect 
to $\Gamma$. Again in the case of the symmetric group, we are led to the "simplex" $\lambda_{1}<\lambda_{2}<\cdots<\lambda_{k}$, which occurs in the work of Karlin and McGregor.

6. Operator formulation. As in the case of [4], the recurrence relations (2.1) possess continuous analogues in the shape of simultaneous Sturm-Liouville problems with several parameters. So far as oscillatory properties are concerned, these have received some investigation [6, pp. 248-251]; the associated orthogonality and eigenfunction expansion were noticed by Hilbert [8, pp. 262-267], who proved the latter in the case $k=2$. There is no need to confine the investigation to systems of equations of the same type; Theorem 1 is readily extended to, for example, recurrence relations coupled parameterwise with, say, partial differential equations. In view of the great variety of such problems, a Hilbert space formulation is clearly called for. Here a somewhat misleading simplicity of the cases mentioned should be observed. In our present case the eigenfunctions are the sequences $p_{n}\left(\lambda^{(\nu)}\right)$, orthogonal in the sense of (4.1), and we note that $p_{n}(\lambda)$ is formed by (2.2), by pointwise multiplication of the solutions of the separate recurrence relations (2.1). With an abstract formulation, such pointwise multiplication must be replaced by a different construction, which we now outline.

The problem (2.1), (2.4) is clearly included in the problem

$$
B_{r} f_{r}+\left(A_{r} \lambda\right) f_{r}=0, \quad r=1, \cdots, k, f_{r} \in H_{r},
$$

where the $H_{r}$ are Hilbert spaces, $B_{r}$ is a self-adjoint endomorphism of $H_{r}, A_{r}=\left(A_{r 1}, \cdots, A_{r k}\right)$ is a row-matrix of such endomorphisms, and $\lambda$ is again a column matrix of scalars. An eigenvalue is again a $k$-tuple $\lambda$ for which (6.1) all have solutions $f_{r} \neq 0$. The corresponding $k$-tuple $\left(f_{1}, \cdots, f_{k}\right)$ may be considered, in the natural way, as an element of the tensor product $\mathfrak{S}=\prod_{r=1}^{k} \otimes H_{r}$. To set up the latter, we introduce firstly a space $\mathfrak{S}^{\dagger}$ containing all finite linear combinations of $k$-tuples

$$
\mathfrak{f}=\sum_{u} \alpha_{u}\left(f_{1 u}, \cdots, f_{k u}\right), \quad f_{r u} \in H_{r}
$$

with scalars $\alpha_{u}$, and for a second such entity

$$
\mathfrak{g}=\sum_{v} \beta_{v}\left(g_{1 v}, \cdots, g_{k v}\right), \quad \quad g_{r v} \in H_{r},
$$

define a scalar product (indicated by a dot), as in [9],

$$
(\mathfrak{f} \cdot \mathfrak{g})=\sum_{u} \sum_{v} \alpha_{u} \bar{\beta}_{v} \prod_{r=1}^{k}\left(f_{r u} \cdot g_{r v}\right),
$$


where $\left(f_{r u} \cdot g_{r v}\right)$ is the scalar product in $H_{r}$. Denoting by $\|\mathfrak{f}\|$ the associated norm in $\mathfrak{S}^{\dagger}$, we complete $\mathfrak{S}^{\dagger}$ by admitting to $\mathfrak{S}^{\dagger}$ convergent series of the form (6.2). If then $\mathfrak{S}_{0}^{\dagger}$ denotes the subset, in fact subspace, of $\mathfrak{f} \in \mathfrak{S}^{\dagger}$ such that $\|\mathfrak{f}\|=0$, we define $\mathfrak{S}$ as $\mathfrak{S}^{\dagger} / \mathfrak{S}_{0}^{\dagger}$, with the same inner product.

Suppose now that $\lambda, \mu$ are distinct eigenvalues, corresponding to eigenelements $\left(f_{1}, \cdots, f_{k}\right)$ and $\left(f_{1}, \cdots, f_{k}\right)$. Forming the scalar product, in $H_{r}$, of (6.1) with $f_{r}^{\dagger}$, and again with $f_{r}, f_{r}^{\dagger}$ interchanged, we have on elimination of $\lambda-\bar{\mu}$ that

$$
\operatorname{det}\left(A_{r s} f_{r}, f_{r}^{\dagger}\right)=0 \text {. }
$$

This determinant may be regarded as a second inner product in $\mathfrak{S}$, with respect to which the eigenelements, considered in $\mathfrak{S}$, are orthogonal; if as in (2.5) it gives a positive-definite form in $\mathfrak{S}$, we can proceed to the reality of the eigenvalues. We may also interpret the eigenvalue equations (6.1) in $\mathfrak{S}$, corresponding to the partial differential or difference equations considered in [4].

Regarding the symmetries considered in $\$ 5$ of this paper, the permutation operators $S$ considered there constitute isometries of $\mathfrak{S}$, commuting with the operators of (6.1). A superficial resemblance will be evident with some of the constructions of [10].

\section{REFERENCES}

1. G. Szegö, Orthogonal polynomials, Amer. Math. Soc. Colloq. Publ. Vol. 23, Amer. Math. Soc., Providence, R. I., 1939.

2. J. Favard, Sur les polynômes de Tchebicheff, C. R. Acad. Sci. Paris 200 (1935), 2052-2053. 1953.

3. A. Erdélyi et al., Higher transcendental functions. II, McGraw-Hill, New York,

4. S. Karlin and J. McGregor, Determinants of orthogonal polynomials, Bull. Amer. Math. Soc. 68 (1962), 204-209,

5. F. V. Atkinson, Discrete and continuous boundary problems, (to appear).

6. E. L. Ince, Ordinary differential equations, Dover, New York, 1944.

7. E. Kamke, Das Lebesgue-Stieltjes Integral, Teubner, Leipzig, 1956.

8. D. Hilbert, Grundzüge einer allgemeinen Theorie der linearen Integralgleichungen, Berlin, 1912.

9. F. J. Murray and J. von Neumann, On rings of operators, Ann. of Math. (2) 37 (1936), 116-229.

10. A. Selberg, Harmonic analysis and discontinuous groups in weakly symmetric Riemannian spaces with applications to Dirichlet series, J. Indian Math. Soc. 20 (1956), 47-87.

UNIVERSITY OF TORONTO 